\title{
EFFECTS OF ALLOPURINOL ON 6-MERCAPTOPURINE THERAPY IN NEOPLASTIC DISEASES
}

\author{
BY \\ R. W. RUNDLES \\ From the Haematology Laboratory, Duke Hospital, and the Department of Medicine, \\ Duke University School of Medicine, Durham, North Carolina, U.S.A.
}

In the first patient treated with allopurinol, in whom remission in chronic granulocytic leukaemia was being produced by the administration of 6-MP as described above, the primary objective of the study $w$ as to determine if the administration of the xanthine oxidase inhibitor would reduce the conversion of 6-MP to thiouric acid (TU). In previous studies, we had found that about 25 per cent. of 6-MP, when given in therapeutic doses, was ordinarily oxidized to thiouric acid (Elion, Callahan, Hitchings, Rundles, and Laszlo, 1962). In the first study, when $150 \mathrm{mg}$. 6-MP was given with $300 \mathrm{mg}$. allopurinol, the formation of thiouric acid was almost completely suppressed. At the same time, there was a 4-fold increase in the amount of free 6-MP excreted in the urine. With $75 \mathrm{mg}$. allopurinol, 10 per cent. of the 6-MP was converted to TU, but the increased amount of 6-MP in the urine remained unchanged. A similar doseresponse effect was obtained in a second patient with chronic granulocytic leukaemia in remission (Table).

TABLE

EFFECT OF ALLOPURINOL (4-HPP) ON CONVERSION OF 6-MP TO 6-THIOURIC ACID (TU) AND EXCRETION OF FREE 6-MP IN THE URINE

\begin{tabular}{l|r|r|r|r}
\hline \multirow{2}{*}{ Cases } & \multicolumn{2}{|c|}{$\begin{array}{c}\text { Dose } \\
\text { (mg.) }\end{array}$} & \multicolumn{2}{c}{$\begin{array}{c}\text { Urinary Excretion } \\
\text { (percentage) }\end{array}$} \\
\cline { 2 - 5 } & 6-MP & 4-HPP & Free 6-MP & \multicolumn{1}{c}{ TU } \\
\hline K.L.C. & 150 & 0 & $7 \cdot 2$ & $25 \cdot 5$ \\
No. F72928 & 150 & 75 & $29 \cdot 5$ & $10 \cdot 0$ \\
C.G.L. & 150 & 300 & $29 \cdot 2$ & $3 \cdot 4$ \\
\hline G.C.J. & 100 & 0 & $3 \cdot 1$ & $24 \cdot 0$ \\
No. 84981 & 100 & 50 & $16 \cdot 8$ & $15 \cdot 7$ \\
C.G.L. & 100 & 200 & $12 \cdot 5$ & $7 \cdot 5$ \\
& 100 & 800 & $27 \cdot 8$ & $2 \cdot 4$ \\
\hline
\end{tabular}

We did not know at that time whether the effective therapeutic dose of 6-MP would be reduced by onequarter, due to the amount salvaged from reduced conversion to thiouric acid, or increased 4-fold in parallel to the augmented urinary excretion of 6-MP, which could indicate that more of the compound was available to circulate in the blood when protected from oxidative degradation.
The effect of allopurinol on the effective therapeutic dose of 6-MP was then studied in seven patients with granulocytic leukaemia and related myeloproliferative diseases (Rundles, Wyngaarden, Hitchings, Elion, and Silberman, 1963). In two patients with untreated chronic granulocytic leukaemia, satisfactory remissions were produced by small doses of 6-MP 150 to $75 \mathrm{mg}$./day, when the compound was given concurrently with 200 to 300 mg. allopurinol.

The effect of allopurinol on the amount of 6-MP required for maintenance therapy was studied in two patients with chronic granulocytic leukaemia, and ino three with related myeloproliferative diseases. In one individual, chronic granulocytic leukaemia haco been satisfactorily controlled for 5 months by the administration of $100 \mathrm{mg}$. 6-MP daily. The effective dose was reduced to $25 \mathrm{mg}$./day, when given concurrently with $100 \mathrm{mg}$. allopurinol. A comparable reduction in therapeutic dose of 6-MP was observed in a second patient who had been controlled on a dose of $100 \mathrm{mg}$. 6-MP daily for a shorter period of time. In the remaining patients with less typical myeloproliferative diseases, the effective therapeutic dose of 6-MP was also reduced by about 75 per cent. by the concurrent administration of 100 to $300 \mathrm{mg}$. allopurinol daily.

As previously reported, there was no evidence of primary haematological effect from the allopurinol itself, other than the occasional production of mild reticulocytosis without evidence of haemolysis (Rundles and others, 1963).

In three of the patients with chronic granulocytic leukaemia, allopurinol was given continuously for periods of several months late in the course of their disease, to see if progression or exacerbation which usually occurs within 2 to 4 years of its onset could be prevented. Unfortunately, the course of disease evolution did not seem to be altered. Each patient had a terminal, acute exacerbation in about the expected period of time.

The important question in reference to the reduc- 
tion of the therapeutic dose of 6-MP produced by allopurinol in patients with leukaemia is whether this means increased therapeutic effectiveness or just dose economy. The administration of allopurinol could conceivably influence the activity of leukaemia by a number of direct or indirect mechanisms: reduction in de novo purine biosynthesis, increase in the amount of purine precursors or derivatives available for re-utilization, increased incorporation of exogenous antipurine compounds, etc.

As a preliminary study in this area, the Southeastern Cancer Chemotherapy Co-operative Study Group undertook a comparison of the anti-leukaemic and haematological effects of 6-MP, and 6-MP plus allopurinol in the treatment of patients with chronic granulocytic leukaemia. The plan of the study is outlined in the Figure. The effect of 6-MP and of 6-MP given with 4-HPP was studied in each patient sequentially. The first regimen was selected at random. The dose of allopurinol was held constant, $300 \mathrm{mg}$. daily, but the dose of 6-MP was adjusted to produce an optimal degree of disease control attained over a period of 3 months. After the initial trial was completed, therapy was suspended to await relapse. A comparable trial of the opposite regimen was then carried out.

A total of 28 patients completed the study. The dose of 6-MP required to produce a given degree of antileukaemic control when administered alone was again about four times that required to produce an equivalent effect when the 6-MP was given with allopurinol. Further analysis of the data showed that the degree of disease control, its lability, the differential haematological effect, time required for the disease to relapse, side-reactions to medications, etc., produced by both regimens was almost exactly the same.
The therapeutic effect of allopurinol in acute leukaemia has been investigated to only a minots. extent. In our own patients a considerable reduc:tion in the prevailing serum uric acid concentrations and in urate excretion has been of no definite antio leukaemic benefit. Vogler and his colleagues haves reported some possible potentiation of the chemo $\overline{\bar{s}}$ therapeutic effect of 6-MP when given with allo purinol (Vogler, Bain, Huguley, Palmer, ando Lowrey, 1966). Further studies of this matter are in progress in co-operative groups.

Even though the major effects produced by $\vec{\omega}$ allopurinol probably have no direct influence on the neoplastic process, the agent has proved to be dis tinctly useful in controlling hyperuricaemic com plications as already described. An elevated serum uric acid level and a great increase in urate excretion occurs frequently in patients with myeloproliferative syndromes, acute leukaemia, plasma cell leukaemial and polycythaemia vera. Gout may be a majoris clinical problem in these patients, too, and whetheer it is primary or secondary in all instances is not easy? to ascertain. In any event, practical therapeuticregimens should provide treatment for the under $Z$ lying disease as well as the associated uric aciof problem.

In recent years aggressive chemotherapeugi regimens have become stylish. This type of manage ment, or the use of irradiation therapy occasionat 8 . may lead to the rapid breakdown of tissue impose some risk of acute, obstructive uric acid nephropathy. Krakoff and Meyer (1965) reporteç that pre-treatment with allopurinol was an effectiv means of preventing hyperuricaemia in patients witlo leukaemia and lymphoma during the early phases $0 \mathbb{B}^{\circ}$ therapy, and virtually eliminated the hazard of urate nephropathy. These findings have been amply con3 firmed by De Conti and Calabresi (1966).

SOUTHEASTERN GROUP PROJECT 205

Influence of HPP on Therapeutic Efficiency of 6-MP

in Treatment of Chronic Granulocytic Leukaemia

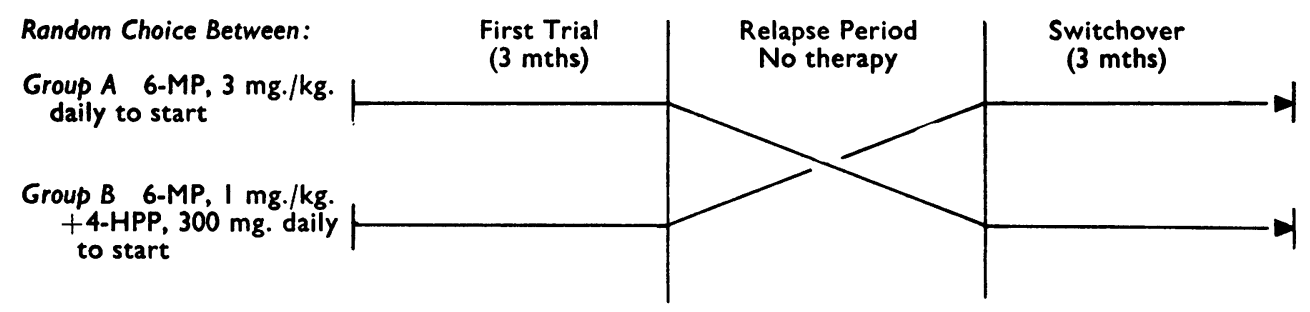

